\title{
A HIGH PERFORMANCE PLANAR PIEZORESISTIVE ACCELEROMETER
}

\author{
Aaron Partridge, J. Kurth Reynolds, Benjamin W. Chui, Eugene M. Chow, Alissa M. Fitzgerald, \\ Lian Zhang, Susan R. Cooper, Thomas W. Kenny \\ Departments of Electrical and Mechanical Engineering, \\ Stanford University, CA 94305-4021
}

\author{
Nadim I. Maluf \\ Lucas NovaSensor, \\ Fremont, CA 94539
}

\begin{abstract}
There is great interest in the use of Deep Reactive Ion Etching (DRIE) to build micromechanical devices. In previous work, we have demonstrated a technique for forming piezoresistive strain gauges on the sidewalls of DRIE high-aspect ratio flexures. In this paper, we report on the development of a planar piezoresistive accelerometer which combines the advantages of simple and inexpensive piezoresistive sensing with the design flexibility offered by DRIE processing of micromechanical structures. The design, fabrication, and experimental testing of these devices is described. The resulting accelerometers offer excellent performance within a technology that should be manufacturable at very low cost. This work also illustrates the potential importance of piezoresistive sensing in DRIE microstructures.
\end{abstract}

\section{INTRODUCTION}

The academic MEMS community has developed a large assortment of accelerometers in the last 20 years, using almost every possible combination of the available transduction mechanisms, materials, and fabrication technologies. Meanwhile, the industrial MEMS community has primarily delivered bulkmicromachined silicon piezoresistive accelerometers. Initially described almost 20 years ago, this simple sensor has proven useful in many applications, is manufacturable at low cost. and does not require sophisticated integration of preamplification circuitry [1]. Commercially successful accelerometers of this sort can be found packaged as hybrids with off-the-shelf instrumentation amplifiers and laser-trimmed thick-film circuits from many companies.

The sensitivity of a piezoresistive accelerometer can be increased by focusing the inertially-induced strain into a very small region. Unfortunately, this strategy reduces the dynamic range of the accelerometer, because there is a maximum strain the silicon flexure can be subjected to before breakage. The anisotropic etching of silicon used to manufacture these parts worsens this problem, because the flexure is always bounded by crystallographic planes. The corners at these intersections cause non-uniformities in the stress, leading to an allowed average strain in the piezoresistor which is well below the maximum that could be tolerated. Rounding of these corners would allow larger values of average strain, and higher sensitivity.

In the last 2 years, many researchers in this community have used Deep Reactive Ion Etching (DRIE) to fabricate high aspect ratio silicon microstructures [2]. This technology allows fabrication of high-aspect ratio planar flexures from single crystal silicon of arbitrary 2-dimensional shape. This is important because it is the first technique suitable for volume fabrication of silicon flexures that is not constrained by the crystal planes. One example application of this technology is the development of planar capacitive accelerometers with crystalline silicon flexures [3].

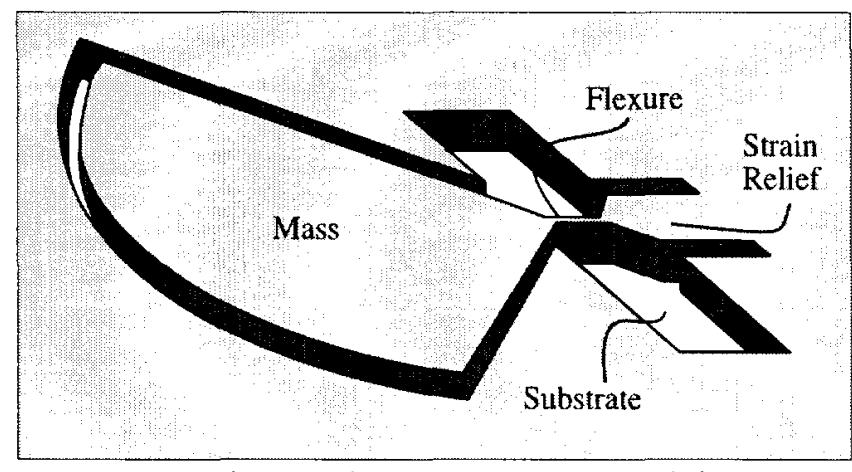

Figure 1 This drawing illustrates the mechanical design of the planar piezoresistive accelerometer. A "pie-shaped" mass is supported from a single high aspect-ratio flexure, which is doped to form a piezoresistive sensor on a vertical surface. The strain relief isolates the strain-sensiive elements of the accelerometer from package-induced stress, and the lateral motion of the mass is caged by the surrounding silicon.

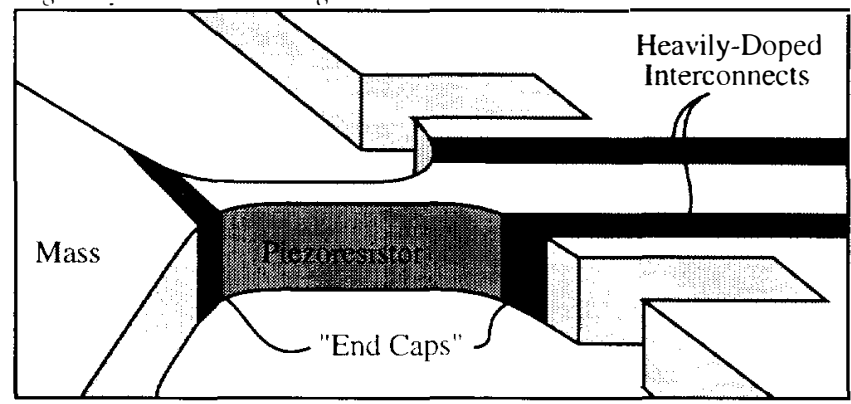

Figure 2. This drawing illustrates a close-up of the flexure, showing the location of the heavily and lightl-doped regions. The electrical circuit path is along the top surface, down the sidewall at the base of the flexure, along one surface of the flexure, back up onto the top of the mass, and back down the backside of the flexure.

In the last year, our group has developed a technique for fabrication of piezoresistive sensors on the vertical surfaces of a DRIE-fabricated planar flexures [4]. This technique uses an oblique ion implantation to form the doped silicon piezoresistors, and opens the door for inexpensive piezoresistive sensing to be used with DRIE-released planar flexures for many applications.

Whenever a flexure and a displacement transducer are combined, the result is an accelerometer. Therefore, it is now relatively easy for researchers with access to ion implantation and DRIE etching to make accelerometers. We set out to demonstrate an inexpensive accelerometer with resolution better than 0.5 $\mathrm{mg} / \mathrm{V}^{\prime} \mathrm{Hz}$, and comparable bandwidth, dynamic range, drift, etc. to the Analog Devices ADXL05, a high-performance. low-cost accelerometer [5] 


\section{DESIGN}

In a piezoresistive sensor, all of the signal-induced strain should be focused into the piezoresistive region, maximizing the resistance change. To achieve this, piezoresistive sensor designers use bosses and other structural elements to concentrate the strain. Building long flexures, as is common in capacitive accelerometer designs, would be detrimental to a piezoresistive accelerometer. Long flexures would decrease the sensors resonant frequency and hence the bandwidth, while spreading the strain over a large volume. For example, the common quad-supported capacitive accelerometer topology is ill-suited to piezoresistive devices because the strain from acceleration is divided over the four legs, each bending sigmoidally, which quarters, then halves the sensor strain. Building piezoresistors on each leg does not win it back: the strain concentration is lost. Therefore, an optimal planar piezoresistive accelerometer should not look anything like a planar capacitive accelerometer. In fact, it really ought to look somewhat like bulk piezoresistive accelerometers, which consist of a mass suspended from one edge by a small flexure.

Guided by this rationale, we have designed and built singly supported proof masses with small piezoresistors on one side of their support flexures as shown in Figs. $I$ and 2.

Assuming that the mass is rigid, the flexure is massless. displacements are small, tangential strain dominates shear strain in the flexure. and the silicon has a simple Young's modulus, we can derive this expression for the strain in the flexure:

$$
\varepsilon=\frac{4 \rho \mathrm{R}^{2} \sin (\theta)}{E w^{2}} \mathrm{~A}
$$

Here, $\rho$ is the density of silicon, $R$ is the length of the proof mass, $\theta$ is the included angle of the proof mass. E is Young's Modulus, the $w$ is the width of the flexure, and $\mathrm{A}$ is the acceleration. Some important conclusions from the equation for strain are that it scales with the cube of the length of the mass, is inversely proportional to the square of the flexure width and is linear with the acceleration. Note that the sensitivity is neither a function of the silicon thickness nor the flexure length.

The sensor bandwidth is limited by the resonant frequency of the proof mass. From the listed assumptions we have a simple spring-mass system with a lateral resonance given by:

$$
f=\frac{1}{2} \pi \sqrt{\frac{E w^{3}}{3 \rho l R^{4}(1+v)(2 \theta+\sin (2 \theta))}}
$$

where $\omega$ is the resonance frequency, $v$ is Poisson's ratio, and $]$ is the flexure length. The lateral resonant frequency is inversely proportional to the square of the proof mass length, to the $3 / 2$ power of the flexure width, and is inversely proportional to the square root of the flexure length. The lateral resonant frequency is not a function of the silicon thickness.

The vertical and torsional resonances are given by:

$$
\begin{aligned}
& f_{\text {cult }}=\frac{1}{2 \pi} \sqrt{\frac{E_{w t^{2}}}{3 \rho l R^{4}(1+v)(2 \theta+\sin (2 \theta))}} \\
& f_{i t u}=\frac{1}{2 \pi} \sqrt{\frac{E w\left(t^{2}+w^{2}\right)}{3 \rho l R^{4}(1+v)(2 \theta-\sin (2 \theta))}}
\end{aligned}
$$

where $t$ is the thickness of the mass and flexure. The out-of-plane resonance is higher than the in-plane resonance by the aspect ratio of the flexure. Simply,
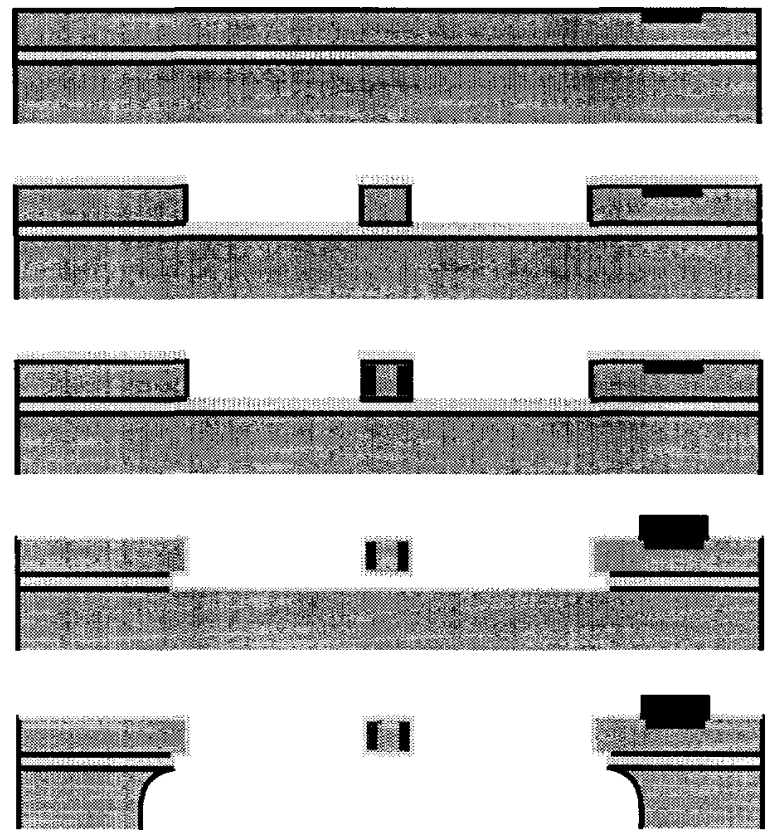

Figure 4. Fabrication procedure. (a) Top-surface implants form $p+$ conductive paths and n+ substrate contacts. (b) Oxides are deposited and the top layer of silicon is etched to define sensors. (c) Oblique implants dope the sensor and return paths on etched sidewalls. (d) Sensors are partially undercut, passivated and bondpads defined. (e) Resist is applied to the topside for protection during release. Backside silicon is plasma etched, and resist is plasma etched, releasing devices.

$$
\frac{f_{c t i t}}{f}=\frac{t}{w}
$$

and there is a similar result for the torsional resonance. From this analysis we can see that we should minimize the flexure width, and maximize the mass length. The narrowest flexure that can be reliably defined in our lithography is about $2.4 \mu \mathrm{m}$. We also built sensors with flexure width of $4.4 \mu \mathrm{m}$. We choose a thickness of 30 microns because of limitations on DRIE undercut control. Sensors were built with length of $0.2 \mathrm{~mm}, 0.5 \mathrm{~mm}, 1.0 \mathrm{~mm}$, and $2.0 \mathrm{~mm}$ to study the variations of yield and performance.

\section{FABRICATION}

We begin with moderately doped n-type wafers, oxidize, bond and grind back to build our BESOI substrate. We next implant and anneal a top surface p+ traces which connect our bondpads to the piezoresistors, and an $\mathrm{n}+$ substrate contact.

Next, we deposit and pattern LTO, and define the structure with a $30 \mu \mathrm{m}$ deep anisotropic silicon etch. After the etch, we implant the sidewalls of the flexure to form the sensor and a return current path. The sensor is moderately $\mathrm{p}$-doped while the opposite side of the flexure is heavily p-doped. This implant is patterned to avoid creating current paths other than through the piezoresistor.

The oxide is etched in HF to undercut the flexure only, and the implants are annealed and passivated with thermal oxide. Then. bond pads are metallized, and the topside is covered in thick resist. The backside is etched in the DRIE to release the parts [6], and the resist is removed in an oxygen plasma. 


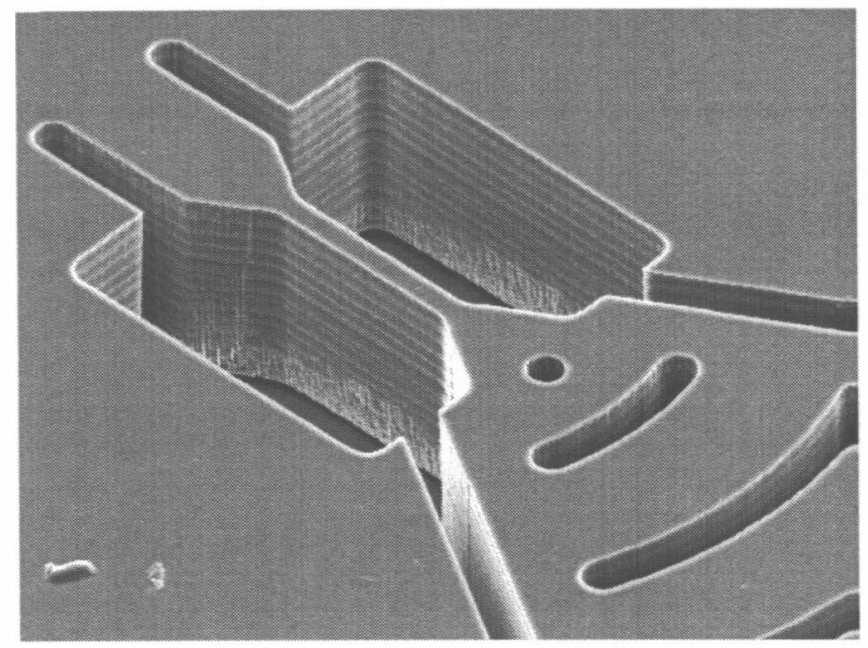

Figure 5 shows an SEM micrograph of the flexure supporting the mass in our accelerometer. This flexure is 30um long and $4.4 u$ wide. The piezoresistors and return current conductors are formed by implanting at approximately $30^{\circ}$ from vertical [4]. The piezoresistor is implanted with medium dose while the return path is implanted with a heavy dose, generating a return conductor. (SEM Phot courtesy of R. Lawton and H. Rockstad, JPL)

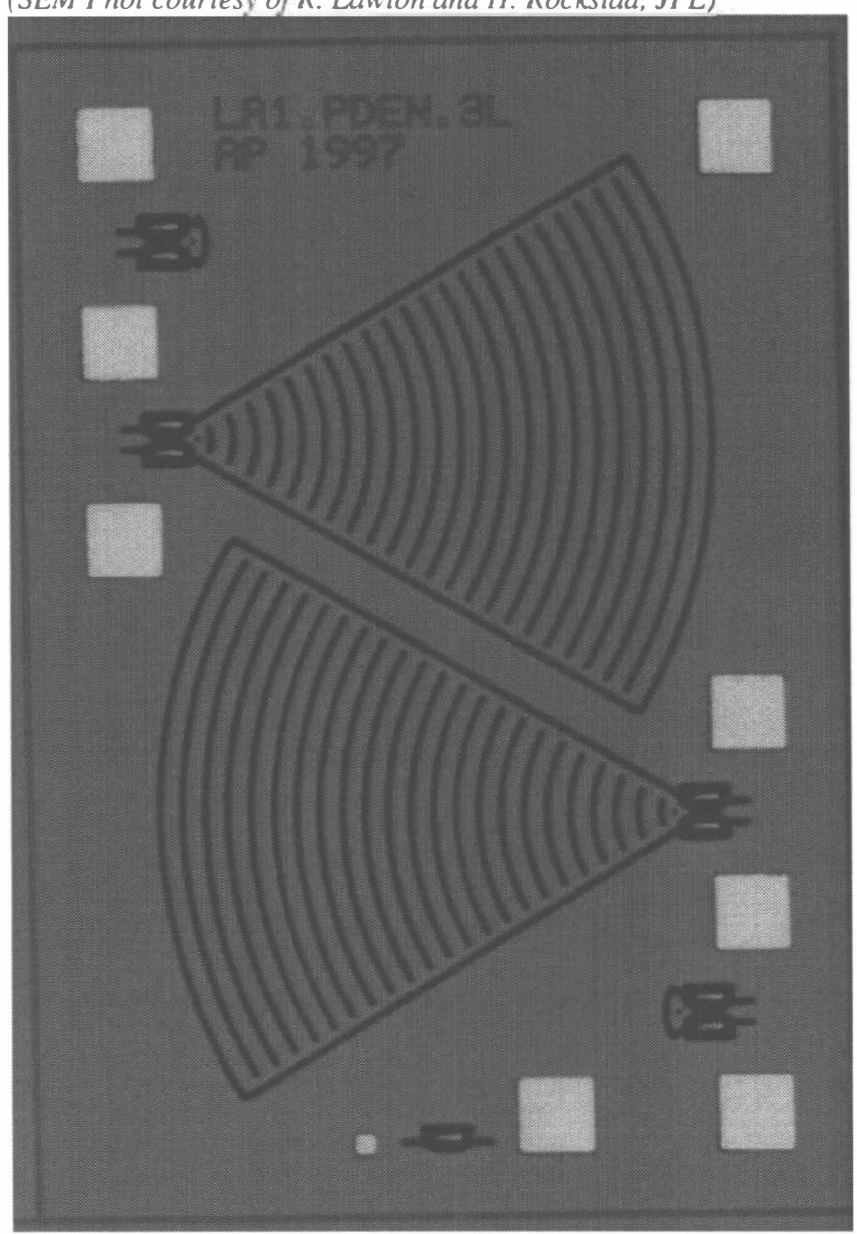

Figure 6 shows an optical micrograph of a complete, full-bridge sensor. The two pie-shaped areas are released proof masses, each joined by a flexure from the tip of the pie to the substrate. Three smaller devices with identical piezoresistors and flexures provide matched bridge resistors and a thermal calibration resistor.

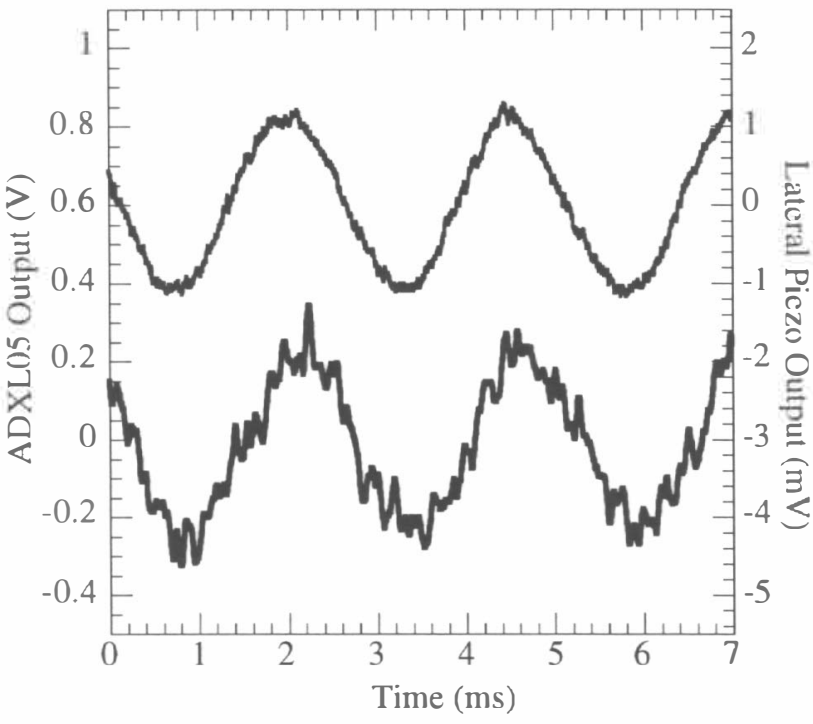

Figure 7. This plot shows the sigle-sweep output signal of a $1 \mathrm{~mm}$ lateral piezoresistive accelerometer (upper curve) and the ADXLO5 (lower curve) to the same $0.2 \mathrm{~g}, 400 \mathrm{~Hz}$ acceleration signal. The signal/noise ratio for the lateral piezoresistor is about $5 x$ better within the full $100 \mathrm{kHz}$ bandwidth of the circuitry used to acquire both signals.

After processing, the devices are mounted in DIPs, and lids are bonded after heating at 150C for 2 hours. The sensors are sealed with air at atmospheric pressure. We believe that this sealing procedure allows some humidity into the package which probably contibutes to surface leakage and adds noise. Experiments on professionally sealed sensors are planned.

\section{EXPERIMENTAL RESULTS}

We have built accelerometers with four proof mass lengths $(0.2 \mathrm{~mm}, 0.5 \mathrm{~mm}, 1.0 \mathrm{~mm}$, and $2.0 \mathrm{~mm})$ and two hinge thicknesses (2.4u and $4.4 \mathrm{u}$ ). We have evaluated these accelerometers for sensitivity, linearity, bandwidth, and noise.

Figure 7 shows the time-domain acceleration response of a $1 \mathrm{~mm}$ lateral piezoresistive accelerometer compared to the response of an ADXL05 accelerometer subjected to the same $0.2 \mathrm{~g}$ acceleration. Both sensors feature mechanical cutoff frequencies near $1 \mathrm{kHz}$, and both are operated with amplification circuits that offer bandwidth in excess of $100 \mathrm{kHz}$. We can see from this data that the lateral piezoresistive accelerometer has a somewhat better signal/noise ratio than the ADXL05 at this frequency.

Measurements of the frequency response of representative lateral piezoresistive accelerometers are shown in Fig. 8. The sensors with the longest masses offer the largest sensitivity, as well as the lowest resonant frequencies. The trends in these parameters with mass length are roughly consistent with expectations from the equations presented earlier. These sensors are damped only by the ambient pressure sealed within the package. There are squeezefilm effects at the ends of the accelerometer motion which can be adjusted by changing the size of the gaps surrounding the mass, or by changing the pressure or viscosity of the gas used to seal these sensors. 


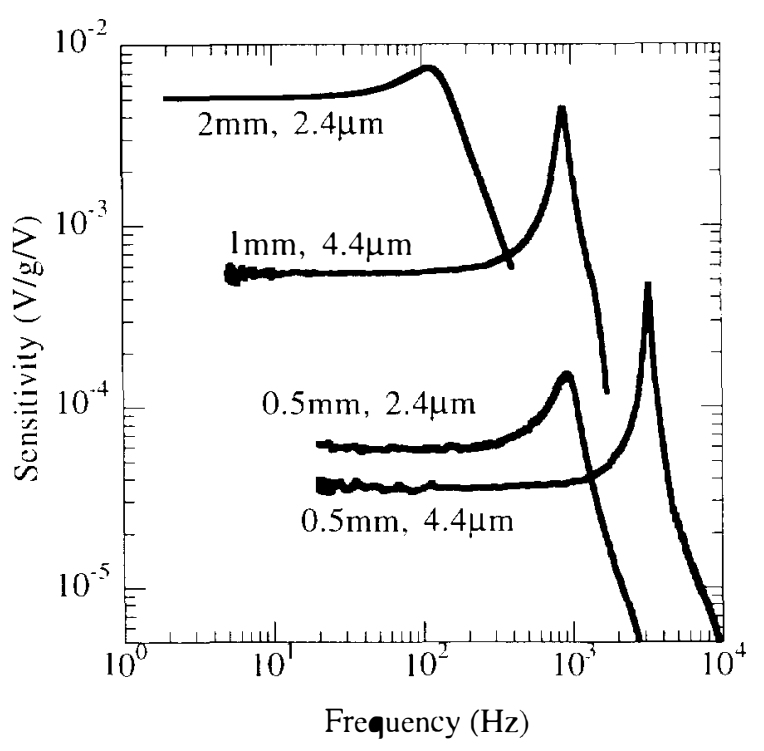

Figure 8. This plot shows the sensitivity of 4 lateral piezoresistive accelerometers as a function of frequency. Each curve is labeled with the length of the proof mass and the width of the flexure.

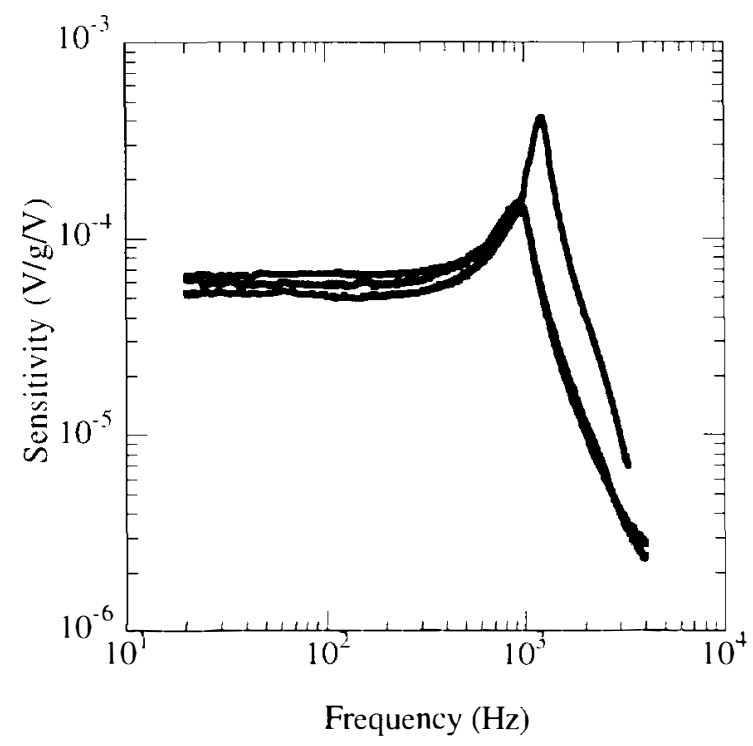

Figure 9. This graph shows the sensitivity of 3 nominally identical $0.5 \mathrm{~mm}, 2.4 \mu \mathrm{m}$ accelerometers to illustrate the uniformity that has been achieved in this process. The sensitivity of these sensors below resonance is within $5 \%$ for this group.

Three different accelerometers with $0.5 \mathrm{~mm}$ flexures were characterized to illustrate the variations in process parameters. Figure 9 shows the measured frequency response of these 3 accelerometers. The variations in sensitivity among these sensors are within $5 \%$ of the mean at all frequencies below the resonance. demonstrating the uniformity possible with the fabrication procedure described above. The sensor with the more pronounced peak was packaged at elevated temperatures in an effort to limit trapped humidity, and is probably at a reduced pressure after cooling. This reduced pressure could explain the increased sensitivity of this device on resonance.

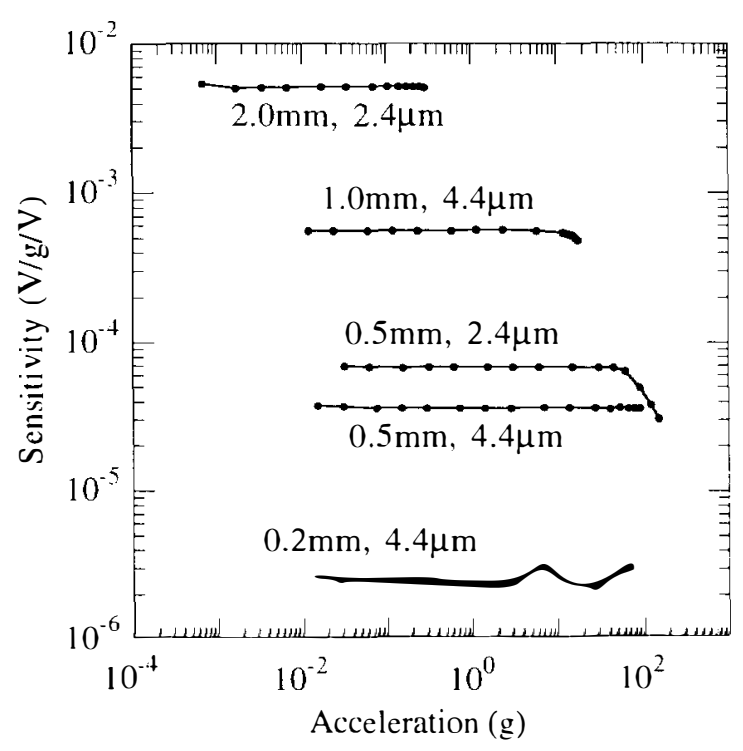

Figure 10. This graph shows the sensitivity of a representative set of accelerometers as a function of applied acceleration signal. In almost every case, the sensors display linearity within $5 \%$ for signals up to the level where the mass begins to undergo collisions with the endstops

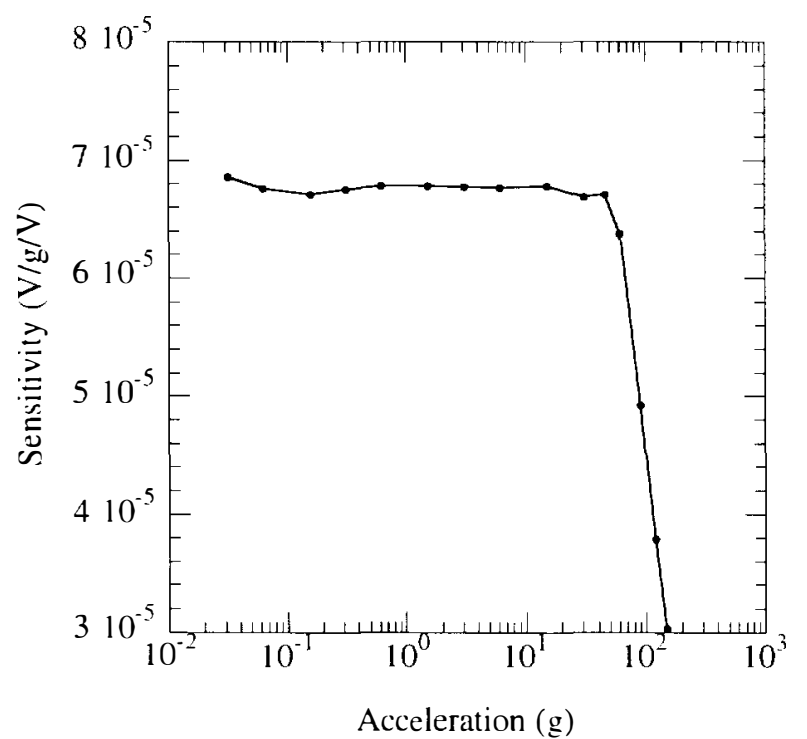

Figure 11. This graph show's the linearity data for the accelerometer with the $0.5 \mathrm{~mm}$ long, $2.4 \mu \mathrm{m}$ wide flexure. The sensor output signal was calibrated against a laser interferometer in this measurement. At signal levels below the collisions with the endstops, the sensitivity was measured to be within $2 \%$ of the mean value.

The linearity of these sensors was measured, and is plotted in Fig. 10. The large $2 \mathrm{~mm}$ sensors are of course the most sensitive but they hit their endstops at the smallest accelerations. The smaller devices have lower sensitivity but greater range. Figure 11 shows the linearity of a typical $0.5 \mathrm{~mm}$ flexure on an expanded vertical scale. This sensor has sensitivity within $2 \%$ of the mean for accelerations measured from $30 \mathrm{mg}$ to $50 \mathrm{~g}$. In all cases. the 
sensors encounter the endstops before significant nonlinearity arises from other sources. These endstops have successfully protected all mounted sensors from breakage without evidence of stiction, but extensive shock testing must be carried out to fully evaluate their performance.

The design of these accelerometers includes a capability for self-test. Electrical contacts can be added to the silicon structures which form the endstops, and a test voltage can be applied to that electrode causing the mass to deflect a known amount. Figure 12 shows a micrograph of an electrode formed at one of the stops which is suitable for this application.

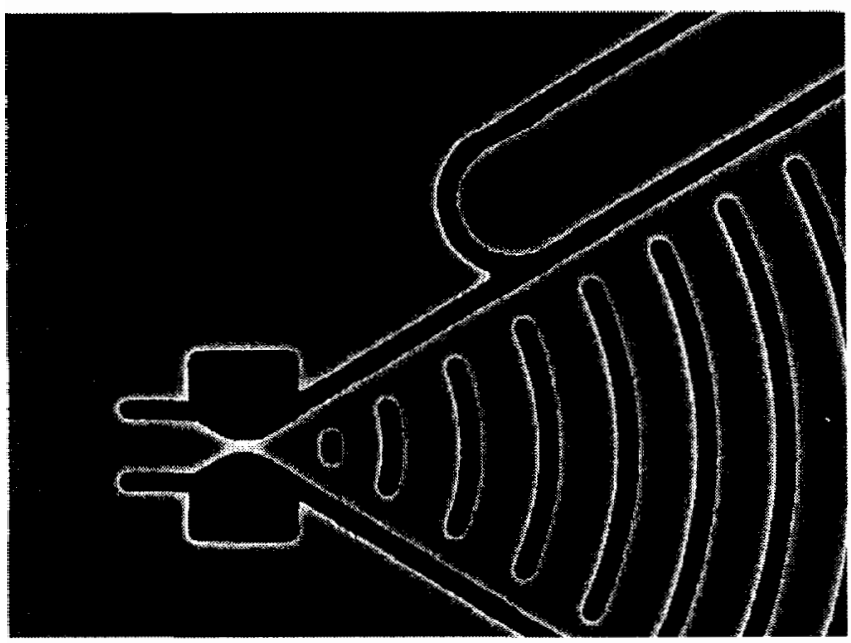

Figure 12. This SEM micrograph show's an image of a lateral piezoresitive accelerometer with additional electrodes for self-test. Voltages may be applied to the element on the upper left of the figure, causing a lateral force on the mass, and producing a suitable acceleration test signal.

Noise in piezoresistive sensors is generally attributed to Johnson noise, amplifier noise, temperature coefficients, and other sources of drift. Most of the real work in improving the performance of commercial piezoresistive sensors is associated with use of temperature compensation and with strain-relieved mounting. Preliminary temperature compensation is achieved by building full Wheatstone bridges using resistive elements with the same temperature coefficients as the sense elements. Strain relief is achieved by "soft die attach" techniques which use compliant materials for mechanical attachment, such as silicone, and minimizing transmission of package stress to the sense elements. We have implemented a full Wheatstone bridge of identical sense elements, as shown in Fig. 6. Strain relief is achieved by attaching the flexure to a mechanical support which is cantilevered off of the substrate, and thereby isolating the strain-sensing elements from substrate stress, as shown in Figs. 1. 2 and 5.

Figure 13 shows measurements of the noise spectra of current "best" and "worst" accelerometers, along with a noise measurement taken from a lateral piezoresistive test structure built in a similar process. In addition, the voltage noise of a commercial piezoresistive sensor is plotted. There are several conclusions to be drawn from this data. First and foremost, the "best" and "worst" accelerometers both exhibit significant noise in excess of the noise measured from a test structure and from the commercial piezoresistive sensor. The source of this excess noise is not precisely known at this time. but it is very likely due to current leakage along the surfaces of the piezoresistor or through the flexure. Work is underway to identify and eliminate this noise source, and there is every reason to believe that the noise can be brought into line with the lowest curves in Fig. 13. Also, the noise from the test structure and the commercial piezoresistive sensor are at about the same level, indicating that the lowest noise curve is a reasonable target for our devices.

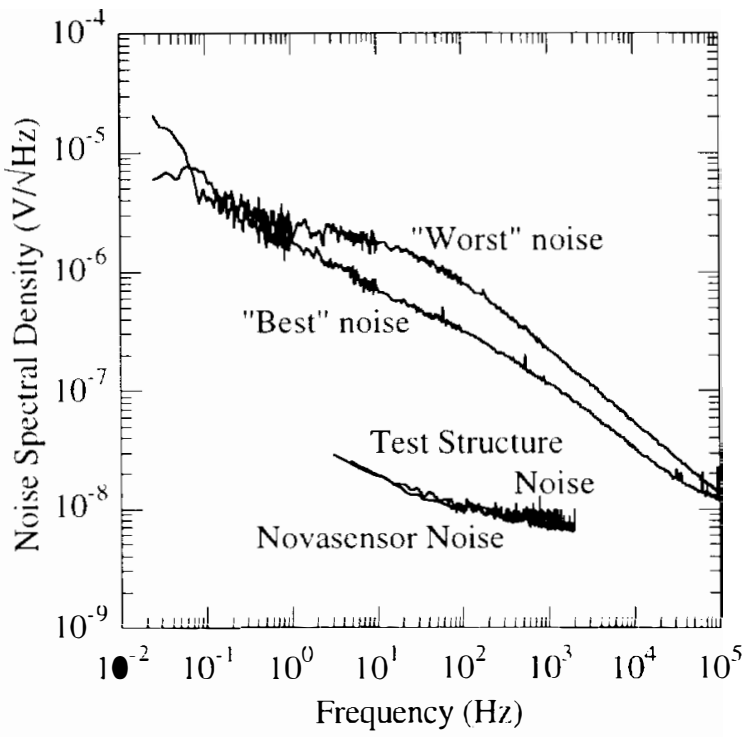

Figure 13. This graph shows the Noise Spectral Density of the lateral piezoresistive accelerometers. The noise is not dependent on size or shape of sensor, but is found to vary between the two upper curves for most sensors in the current generation. For comparison, we also plot the noise of a commercial piezoresistive pressure sensor, and a lateral piezoresistive test structure made in a process very similar to the accelerometer process [4].

Given the sensitivity curves shown in Fig. 8 and the noise curves in Fig. 13, we can plot the resolution of our lateral piezoresistive accelerometers, as shown in Fig. 14. This data shows that the current performance of these sensors is already competitive with the ADXL05. It is important to note that the performance of these sensors should improve significantly if the noise can be lowered to the levels that have been measured for lateral piezoresistive test structures.

The performance of these accelerometers is summarized in Table 1. This table compares the $1 \mathrm{~mm}$ lateral piezoresistive accelerometer to the commercial specifications for the ADXL05. and indicates that the current performance of this device is already very impressive.

$\begin{array}{lll}\text { Parameter } & \text { ADXL05 } & 1 \mathrm{~mm} \text { Lateral Piezoresistor } \\ \text { Sensitivity } & 200 \mathrm{mV} / \mathrm{g} & 0.7 \mathrm{mV} / \mathrm{g} \\ \text { Bandwidth } & 1.5 \mathrm{kHz} & 1 \mathrm{kHz} \\ \text { Range } & 5 \mathrm{~g} & 10 \mathrm{~g} \\ \text { Linearity } & 0.2 \% & <2 \% \\ \text { Noise } & 0.1 \mathrm{~g} \mathrm{RMS} & 0.03 \mathrm{~g} \mathrm{RMS} \\ \text { Resolution } & 0.5 \mathrm{mg} / \mathrm{v} \mathrm{Hz} & 2 \mathrm{mg} / \mathrm{V} \mathrm{Hz}-0.04 \mathrm{mg} / \mathrm{v} \mathrm{Hz}\end{array}$

Table 1 Comparison between ADXLO5 and present performance for $1 \mathrm{~mm}$ lateral piezoresistive sensor: 


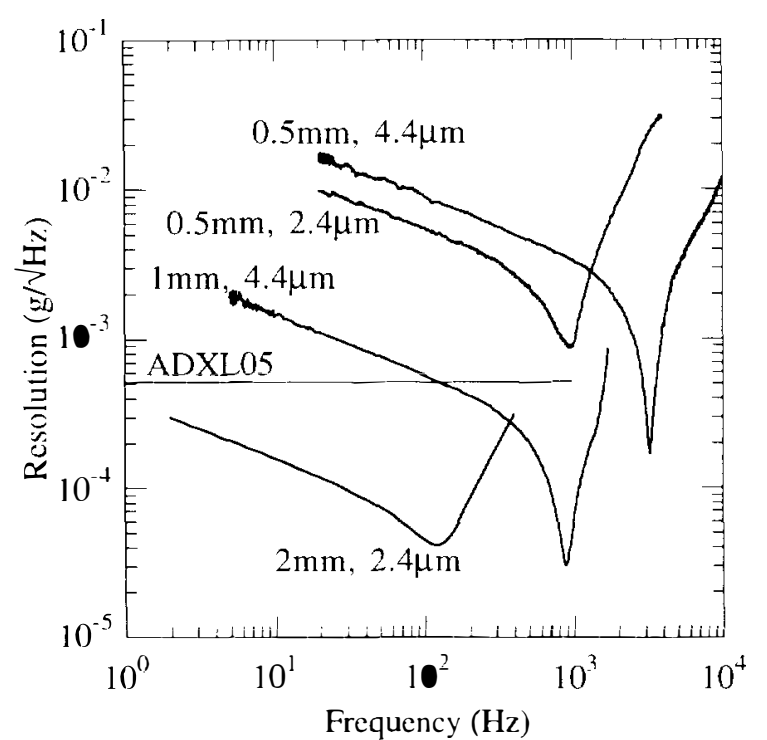

Figure 14. This graph shows the resolution of several lateral piezoresistive accelerometers. The resolution of the ADXLO5 is shown for reference

\section{DISCUSSION}

Piezoresistive sensing offers many important advantages for low-cost sensors. Probably the most significant advantage is that the resistance measuring circuitry can be implemented as low-cost hybrids with laser-trimmed thick-film circuit elements for offset compensation. Many companies have manufactured small, moderate, or large quantities of such sensors while maintaining profit margins in low-cost applications. Clearly, any measurement technology which can capitalize on this infrastructure and improve performance should find broad applications.

The piezoresistive sensors described here can be inserted directly into such a production environment. The fabrication process used for these sensors was developed in close coordination with Lucas NovaSensor, and is specifically compatible with existing low-cost sensor fabrication procedures in use in their facility. Reliable cost models also exist at Novasensor for sensors manufactured using these techniques, and these models indicate that the sensors described in this manuscript can be manufactured in moderate quantities for a few dollars/unit.

The current performance of these sensors is comparable with ADXL05 performance levels. The limitation to this performance is currently due to excess noise related to surfacc or internal leakage currents in the sensor. We have good reason to believe that this noise source can be eliminated, because it has been eliminated in test structures fabricated using the same approach. If this noise can be brought into agreement with the noise seen on test structures, the resolution of this sensor will improve to as much as 2-3 orders of magnitude below that of the ADXL05, which would enable use of this sensor in a much broader range of applications.

Generally, the academic MEMS community has not shown much recent interest in piezoresistive sensing methods. The wellknown problems with temperature coefficients and limited sensitivity have probably contributed to this lack of interest. Nevertheless, industrial manufacturers of piezoresistive sensors have managed to handle these problems with careful packaging and bridge circuits. Given the higher cost of other sensing technologies, perhaps piezoresistors deserve some re-examination. The combination of piezoresistive sensing and DRIE-etching of planar flexures may have many applications beyond acceleration sensing, such as gyroscopes, flow sensors, magnetic field sensors, and so on.

\section{CONCLUSIONS}

We have designed and built a high-performance piezoresistive accelerometer using DRIE etching and oblique ion implantation. The resulting prototype accelerometers offer performance parameters consistent with the best inexpensive commercial accelerometer, the ADXL05. and there is good reason to expect significant performance improvements very soon. Finally. this general technology may find broad use in other sensing applications.

\section{ACKNOWLEDGEMENTS}

The authors thank Dan Rugar of IBM Almaden for advice and assistance on critical point drying, Pauline Prather of Stanford for assistance with device packaging, and Howard Rockstad of JPL for support and patience. This work is supported by JPL/NASA, NSF CAREER (ECS-9502046), NSF Instrumentation for Materials Research (DMR-9504099), and the Terman Fellowship.

\section{REFERENCES}

1. L.M. Roylance and J.B. Angell, "A Batch Fabricated Silicon Accelerometer", IEEE Transactions on Electron Devices, ED-26, 1911 (1979).

2. For examples, see E.H. Klaassen et.al, "Silicon Fusion Bonding and Deep Reactive Ion Etching: A New Technology for Microstructures". 1995 International Conference on Solid-State Sensors and Actuators. (Transducers '95) 556 (1995).

3. B.P. van Drieënhuizen, N.I. Maluf, I.E. Opris, G.T.A. Kovacs, "Force-Balanced Accelerometer with $\mathrm{mG}$ Resolution, Fabricated using Silicon Fusion Bonding and Deep Reactive Ion Etching" 1997 International Conference on Solid-State Sensors and Actuators. (Transducers '97), 1229-30, 1997.

4. B.W. Chui, T.W. Kenny, H.J. Mamin, B.D. Terris, and D. Rugar, "Independent Detection of Vertical and Lateral Forces with a Sidewall-Implanted Dual-Axis Piezoresistive Cantilever", Appl. Phys. Lett. 72. 1388 (1998). and B.W. Chui, H.J. Mamin, B.D. Terris, D. Rugar. T.W. Kenny, "Sidewall-Implanted Dual-Axis Piezoresistive Cantilever for AFM Data Storage Readback and Tracking.", Proceedings of IEEE International Workshop on MEMS. Heidelberg, pp. 12 (1998).

5. F. Goodenough. "Airbags Boom when IC Accelerometer Secs 50g”, Electronic Design 39 (1991). and Analog Devices ADXL05 Data Sheet, Analog Devices, Norwood CT.

6. E.M. Chow et al. "Fabrication of High-Density Cantilever Arrays and Through-Wafer Interconnects", Proceedings, 1998 Solid State Sensor and Actuator Workshop. Hilton Head. this digest (1998). 